\title{
Les TICE au Maroc Entre instauration technologique et conduite de changement, cas des établissements scolaires
}

\begin{abstract}
Khadija Raouf,
Professeur de l'Enseignement Supérieur Habilité en didactique des sciences Centre Régional des Métiers de l'Education et de la Formation Casablanca-

Settat Groupe de Recherche Interdisciplinaire sur les Systèmes d'Enseignement Apprentissage des Sciences et Techniques, Maroc
\end{abstract}

\section{Amina Boulahoual,}

Professeur de l'Enseignement Supérieur Assistante Centre Régional des Métiers de l'Education et de la Formation Rabat-Salé-Kénitra Laboratoire de Recherche Scientifique et Innovation Pédagogique, Maroc

\section{Fatima Zahra Mezzat,}

Professeur d'informatique, Direction provinciale Anfa., Casablanca, Maroc

\section{Meriem Sahli,}

Professeur de l'Enseignement Supérieur Assistante, Université Al Akhawayn, Ifrane, Maroc

\section{Résumé}

Des résultats d'études relatives à l'intégration des technologies d'information et de communication pour l'Enseignement (TICE) au Maroc révèlent un taux d'intégration en deçà des objectifs escomptés malgré un contexte institutionnel favorisant la généralisation des TICE. Partant de ces constats, les travaux de cette recherche ont été axés sur l'analyse des rapports personnels des enseignants marocains aux TICE dans un cadre de conduite de changement. Avant de caractériser lesdits rapports, il a été nécessaire de procéder d'abord à l'analyse des documents officiels puis ceux des résultats de recherches antérieures, alimentés par ceux de dépouillement du questionnaire que nous avons élaboré et soumis à des enseignants volontaires de différents cycles et régions du Maroc. Les principaux résultats semblent en faveur d'une vision floue en lien avec le programme GENIE ou méconnue de certains répondants et l'incidence des réformes promues sur les pratiques enseignantes demeure timide malgré les efforts louables de généralisation.

Mots clés : TICE, Pratiques enseignantes, Changement, GENIE 


\title{
ICT in Moroccan Schools Between Technological Implementation and Change Leading
}

\begin{abstract}
Khadija Raouf,
Professeur de l'Enseignement Supérieur Habilité en didactique des sciences Centre Régional des Métiers de l'Education et de la Formation Casablanca-

Settat Groupe de Recherche Interdisciplinaire sur les Systèmes

d'Enseignement Apprentissage des Sciences et Techniques, Maroc
\end{abstract}

\section{Amina Boulahoual,}

Professeur de l'Enseignement Supérieur Assistante Centre Régional des Métiers de l'Education et de la Formation Rabat-Salé-Kénitra Laboratoire de Recherche Scientifique et Innovation Pédagogique, Maroc

\section{Fatima Zahra Mezzat,}

Professeur d'informatique, Direction provinciale Anfa., Casablanca, Maroc

\section{Meriem Sahli,}

Professeur de l'Enseignement Supérieur Assistante,

Université Al Akhawayn, Ifrane, Maroc

\begin{abstract}
Previous research findings revealed that integration of information and communication technology (ICT) in schooling is below expectations even though the institutional context promotes the use of ICT. Based on this, the purpose of this study is to investigate the relationship Moroccan teachers have to the use of ICT by adapting leading change approach. To do this, we first analyzed official documents and speeches and results of previous studies. Also, we studied the results of the questionnaire that we developed and distributed to teachers from different school levels and areas. The results of this study show that some teachers have a blurred vision of the ICT project 'GENIE' and some of them ignore what this project is about. This investigation concluded that reforms to integrate ICT in schooling remain very limited despite efforts to spread the use of technology in the Moroccan school.
\end{abstract}

Keywords: ICT, Teaching practices, Change, GENIE

\section{Introduction}

L'institution Marocaine chargée de l'éducation et de la formation se focalise de plus en plus sur l'intégration des Techniques d'Information et de la Communication (TIC) dans les pratiques enseignantes par l'équipement des 
établissements scolaires, l'instauration d'offres variées de programmes, dispositifs et centres de formation.

En effet, la Stratégie Maroc Numéric 2020 (UNESCO,2017) vient renforcer celle de 2013 (MICNT, 2009) qui se déclinait en quatre priorités stratégiques où les acteurs de l'enseignement occupent une place importante dans l'axe transformation sociale et trace l'usage des TICE parmi ses objectifs principaux. Ces Stratégies Maroc Numéric ont permis de donner un nouveau souffle au programme GENIE, qui est un programme national pour la généralisation des Technologies d'Information et de Communication dans l'Enseignement (TICE) au Maroc. Celui ci prône le renforcement des compétences des enseignants et la promotion de l'usage des ressources numériques en classe pour les différents cycles afin d'améliorer les apprentissages et assurer le développement professionnel des enseignants.

Or, comme 'l'innovation est un processus qui a pour intention une action de changement et pour moyen l'introduction d'un élément ou d'un système dans un contexte déjà structuré" comme le rapporte CROS (2004, page 19), ce genre de réformes exige une prise en compte du contexte de changement de type organisationnel, structurel et culturel dans le processus d'intégration afin que les enseignants, les directeurs des établissements et tous les intervenants se sentent soutenus, accompagnés et non subissant les changements, en particulier ceux qui ont une vision désabusée de l'usage des TICE (Janner et al, 2013). En effet, l'introduction des technologies d'information et de communication dans les établissements scolaires non préparés à cet effet influencera peu les pratiques des enseignants et les résultats scolaires (Sandholtz et al, 1997; Isabelle et al, 2002).

Certes des études sur l'intégration et l'utilisation des TICE ont été réalisées par plusieurs chercheurs marocains (Messaoudi et Talbi, 2012; El Ouidadi, 2012; Mastafi, 2013; El Madhi et al, 2014; Benheddi, 2015) mais depuis, qu'en est-il surtout avec le contexte de la nouvelle stratégie de généralisation des TICE au Maroc et l'implication des politiques dans le projet? Ces efforts louables ont été effectivement salués récemment par le conseil supérieur de l'éducation, de la formation et de la recherche mais qu'en est-il sur le terrain ? Sur les pratiques enseignantes, les représentations des enseignants vis à vis de la vision élaborée ? et puis outre l'importance d'une vision partagée, sa mise en œuvre exige, entre autres, de doter les acteurs de moyens sur le plan développement et renforcement de compétences, équipements et infrastructure. Qu'en pensent les acteurs principaux ?

Partant de ces questionnements, cette recherche s'est intéressée à la problématique liée aux rapports personnels des enseignants à l'objet TICE afin d'essayer d'explorer l'incidence des réformes promues au niveau des établissements scolaires auxquels les répondants sont rattachés, sur les 
pratiques enseignantes déclarées et leur représentations relatives aux changements induits.

\section{Revue de Littérature}

Avant les années 60, l'expression médias d'apprentissage ou médias d'enseignement était d'usage pour désigner appareils et instruments utilisés à des fins d'enseignement. Plus tard, le terme technologies commença à se répandre dans le champ éducatif pour les désigner. Le terme NTE (Nouvelles Technologies d'Enseignement) paru après pour être remplacé plus tard par le terme TICE (Basque,2006) qui est l'acronyme de Technologie d'Information et de Communication pour l'Enseignement ou l'Education. Les TIC est un ensemble assez étendu de Technologies d'Information et de Communication, cet ensemble regroupe des machines, des logiciels et des services de toutes sortes. Les TIC incluent, entre autres, les technologies liées à l'Internet, les multimédias diffusés sur cédérom, la robotique, la réalité virtuelle, etc.

$\mathrm{Au}$ Maroc, plusieurs actions et projets, visant l'intégration des TICE, virent le jour depuis l'initiation du Réseau MARWAN en 1998 (MAP, 2009), suivi chronologiquement par la Charte Nationale d'Education et de Formation, spécialement le levier 10 en 1999 (MEN,2019), le Campus Virtuel Marocain en 2004 (Sidir, 2008), le Concours des Enseignants Innovants depuis 2005 (Ahaji,2010), le Programme GENIE qui fut lancé en 2006 puis révisé en 2008 (Abouhanifa, 2009), les programmes Nafida, Injaz et Lawhaty (Nafidi, 2018), le Portail National Taalimtice 2011 (GENIE, 2009), le Plan Maroc Numeric 2013 (MCINET, 2009), le Plan Maroc Digital 2020 et La Vision Stratégique Pour la Réforme de L'école 2015-2030 (CSEFRS) pour n'en citer que ceuxci.

Toutefois, toute innovation produit ou induit un changement comme le rapporte Cros (2004), Moscovici (1979) précise que tout changement n'est pas nécessairement innovation alors que toute innovation se manifeste par un changement, qui est donc la facette visible et observable conséquente à "la création ou à l'appropriation de la nouveauté". Ce changement constaté peutêtre aussi bien en accord avec l'effort fourni pour instaurer la nouveauté qu'en dissonance, en fonction d'un ensemble de facteurs qui affectent la réussite de la conduite du changement. Ainsi, l'ampleur des efforts déployés par l'état et les constats relevés du terrain poussent davantage à questionner la dimension relationnelle aux technologies, au-delà de l'usage et des conditions d'usages, vu que l'appropriation des TICE par les enseignants ne saurait se parfaire sans une réflexion sur les enjeux et donc la vision globale, sur les conséquences épistémologiques et cognitives des outils ainsi que sur les rapports personnels qui les lient à ces technologies.

En 2012, une étude réalisée à l'Académie Régionale de l'Education et de la Formation de Meknès-Tafilalet (Messaoudi et Talbi, 2012) a touché les 
1500 acteurs éducatifs concernés par la caravane TICE et a permis de lister un ensemble de contraintes, allant de la sous-exploitation des équipements existants, au manque de développement professionnel du corps enseignant, passant par une gestion locale défaillante, par les problèmes logistiques des formations jusqu'aux difficultés liées au suivi et à l'encadrement des formations au niveau régional et local, sans oublier l'insuffisance des contenus pédagogiques numériques validés (Messaoudi, Talbi, 2012). Des contraintes assez similaires ont été soulevées par une autre enquête datant de la même année (El Ouidadi, 2012), l'auteur ajoute la présence d'attitudes négatives des enseignants qui paraissent satisfaits des outils traditionnels $(10.7 \%)$ ou accordent un intérêt trop faible aux TICE (26.5\%) ou expriment une incertitude quant à leur intérêt en classe (13\%), allant même jusqu'à adopter une opposition de principe contre les TICE (7.5\%). Plus tard en 2013, une enquête auprès de 832 enseignants du primaire et du secondaire appartenant à l'académie de Doukkala-Abda (Mastafi, 2013) vient confirmer ce constat en concluant que l'usage des TICE dans le système éducatif Marocain reste encore très limité, voire même absent de la pratique de la plupart des enseignants enquêtés. Une autre contrainte vient s'ajouter à la liste, celle de l'interdiction d'accès aux salles multimédias, quand elle existe, par le directeur de l'établissement scolaire par crainte de détérioration du matériel (Benheddi, 2015), sachant que le leadership de la direction est un des facteurs qui affecte considérablement l'utilisation des technologies (Isabelle et al, 2002). Ces constatations n'émanent pas uniquement du contexte marocain, une enquête réalisée en France auprès des enseignants du primaire va en effet dans le même sens mais de façon certes moins accentuée, l'auteur atteste que l'outil favorise la motivation des apprenants et facilite l'apprentissage mais recense des problèmes aussi bien matériels, de configurations, organisationnels que pédagogiques et souligne tout aussi la présence persistante d'une certaine cristallisation des valeurs des enseignants autour des TICE (Hamon, 2012).

Ces constats révèlent que les réformes promues en lien avec l'infrastructure, la formation et les contenus numériques ne sont pas perceptibles par une grande partie des sujets. En effet, on note des contraintes liées à la résistance au changement des pratiques pédagogiques qui semblent émaner du manque de motivation extrinsèque et à la non maîtrise de l'usage pédagogique des TICE et celles relatives à l'infrastructure comme les équipements, les logiciels et la connexion à internet (Messaoudi et Talbi, 2012; Mastafi, 2013; El Madhi et al, 2014; Benheddi, 2015) ainsi que celles en lien avec la politique et la stratégie de mise en œuvre des TICE que la majorité des enseignants ayant participé à l'enquête réalisée par Mastafi estime qu'elles manquent de clarté et de cohérence. Ce qui expliquerait éventuellement cette résistance au changement de pratiques des enseignants. 
Champagne (2002) affirme qu'aucun modèle ne permettrait de "prédire dans quelles conditions se manifestera la résistance et quels processus d'implantation permettraient de les contrer", toutefois, certains auteurs mettent en avant le côté psychanalytique relatif aux mécanismes de défense, d'autres la peur de perdre des acquis alors que certains l'attribuent à la personnalité (Kets de Vries et Miller, 1985; Scott et Jaffe, 1992; Collerette, Delisle et Perron, 1997, cités par Champagne, 2002). Quelques soit les facteurs, Nimier (2016) conditionne le changement réussi, entre autres, par la clarté de la vision globale en précisant que le changement doit s'expliquer pour assurer un cadre rassurant et éviter d'engendrer "une polémique où chacun se bat contre des moulins à vent", ainsi que par la nécessité de discuter le changement avec les intéressés vu l'impossibilité de cerner toute sa complexité, et ce afin de découvrir des besoins que les responsables de l'instauration du changement n'auraient éventuellement pas considérés comme primordiaux alors qu'ils le seraient pour les intéressés ou qu'ils n'auraient carrément pas pris en compte au risque de voir des résistances s'accentuer et causer des effets pervers opposés à l'attendu. L'aide efficace est tout aussi fondamental, le changement peut entraîner "une perte de compétence, [...], et une tension nerveuse étant donné que la part de l'incertain prend le pas sur celle de l'incontestable" (Ducros, 1981, p.103, cité par Nimier, 2016), ce qui impose qu'en plus de mettre à disposition "une aide pratique, il est extrêmement important d'apporter au professeur un solide soutien psychologique" (ibid).

Le modèle de changement de Kotter (1996) insiste aussi via ses 8 étapes, sur l'importance de la vision comme pilier du changement. Il part en effet du fait que les changements résident davantage dans la méthodologie suivie via un bon dispositif de communication, de formation et d'accompagnement que dans le projet lui-même. L'ancrage de nouvelles approches dans la culture de l'organisation nécessite selon le modèle de Kotter, trois phases intégrant 7 sous processus du changement. La première phase préparatoire relative à la création d'un climat propice au changement devrait se faire en trois sous processus: Création d'un sentiment d'urgence justifiant le changement, la formation d'une coalition et enfin le développement de vision et de stratégies.

La mise en œuvre de cette vision se base essentiellement sur l'engagement et la délégation de pouvoirs en communiquant autour d'elle, en incitant à l'action et en partageant les résultats réalisés à court terme pour plus de motivation. La troisième phase, caractérisée par l'instauration du nouveau équilibre et donc une nouvelle culture, est une phase d'implantation du changement et de consolidation. Pour ce faire, il faut accompagner, former et donner les moyens permettant de garantir la cohésion et l'adhésion au projet de changement. Minvielle et Contandriopoulos (2004) listent à leur tour 8 
descripteurs du changement vu comme processus social qui a lieu dans un contexte donné, et dont il faudrait tenir compte avant de l'initier pour garantir sa réussite. Parmi ces descripteurs, se trouve encore la condition de clarté des grands enjeux du changement et par conséquent de sa vision globale ainsi que l'identité des acteurs, l'environnement dont l'observation de sa concordance avec le changement peut le favoriser comme le freiner, organisationnel, physique ou culturel soit-il.

Si les auteurs sont d'accord concernant la nécessité de la clarté de la vision et de l'implication des acteurs, la lenteur de l'instauration des pratiques basées sur les technologies dans le contexte scolaire pourrait elle-être due à une ambiguïté chez les enseignants, voire même une méconnaissance de la vision globale de ce changement? Pourrait-elle être due à la faible prise en considération des acteurs lors de l'instauration? Ces rapports personnels des enseignants à l'objet TICE seraient-ils liés à un manque et/ou à la qualité faible de la formation et des moyens nécessaires à la conduite de changement dans la pratique des enseignants ? Les nouveaux enseignants seraient-ils avantagés vu leur sensibilisation aux technologies via le module TICE aux Centres Régionaux des Métiers de l'Education et de la Formation (CRMEF)?

\section{Elements Methodologiques}

\section{Site de l'étude:}

L'étude a été réalisée au Maroc en décembre 2018 et a concerné les milieux urbains, ruraux et semi-urbains.

\section{Population et échantillon}

Cette étude a ciblé les enseignants marocains en exercice, de différentes disciplines, différents cycles et milieux (rural et urbain) et ce à l'échelle nationale. Pour l'échantillon, il est constitué de 433 enseignants ayant répondu volontairement au questionnaire.

\section{Instruments de collecte des données}

Afin de dégager les rapports personnels des enseignants aux TICE dans une optique de conduite de changement et tenter d'évaluer l'incidence des réformes sur leurs pratiques, un questionnaire a été conçu via le logiciel de questionnaire en ligne Drag'n Survey et diffuser via les réseaux sociaux en ciblant particulièrement les groupements d'enseignants marocains. Ce logiciel permet la création, la diffusion et l'analyse des données.

Le questionnaire est composé de 14 items de type fermés et semi ouverts et il couvre :

- les données personnelles des répondants

- l'usage des TICE

- la connaissance du programme GENIE 
- l'infrastructure et l'équipement des établissements y compris l'offre de contenus numériques éducatifs et pédagogiques en lien avec les curricula et

- l'accompagnement des enseignants dans l'intégration des TICE. En particulier le volet formation.

Les données personnelles renseignent entre autres, sur le milieu (rural? urbain? semi urbain?) et l'ancienneté dans l'enseignement. La variable concernant le milieu de travail a paru importante vu que le milieu rural pourrait être moins équipé que le milieu urbain. Quand à l'ancienneté, les centres de formation des enseignants (CRMEF) a connu une réforme au cours de la dernière décennie qui a touché notamment au domaine des TICE. Les enseignants stagiaires bénéficient depuis cette réforme d'un module de formation dédiée à l'usage de technologies en éducation et par conséquent, la variable ancienneté permettrait de vérifier si ladite formation avait impacter l'usage.

Pour les autres items, les variables relèvent d'une part, d'éléments clé pour une conduite de changement efficace tels que la communication sur le programme GENIE, le renforcement des compétences des acteurs et la dotation des établissements d'équipements matériels et numériques. D'autre part, des 3 axes majeurs retenus, sur le plan institutionnel, pour l'introduction efficace des TICE à savoir l'infrastructure par l'aménagement de salles multimédia dans tous les établissements, l'offre de contenus numériques éducatifs et pédagogiques en lien avec les curricula et l'accompagnement des enseignants dans l'intégration des TICE en fonction de leurs besoins professionnels.

\section{Mode de traitement des données.}

Les 433 questionnaires collectés ont été analysés d'abord par le logiciel Drag'n Survey. Les données recueillies ont été ensuite traitées via le logiciel Microsoft Excel. La création de divers tableaux croisés a permis de mettre en avant les corrélations existantes entre les variables en lien avec les caractéristiques de la population cible, les éléments clé pour une conduite de changement efficace et les axes retenus pour l'introduction efficace des TICE susmentionnés. Les documents officiels relatifs à l'intégration des TICE au Maroc (Stratégies, rapports d'évaluation interne, bilans du programme GENIE et projet E1P10, guides d'intégration des TICE, etc.) ont été pris en compte dans l'analyse des données recueillies afin de mieux caractériser les rapports personnels des enseignants à l'objet TICE.

Eu égard de l'incidence de ces réformes sur les pratiques enseignantes et la culture existante, la conduite du changement a été adoptée comme approche d'analyse. Pour ce faire, des éléments clé pouvant favoriser le 
changement, ont été pris en considération tels que la communication sur le programme génie, la qualification et le renforcement des compétences des acteurs principaux à savoir les enseignants et la dotation des établissements scolaires en équipements et infrastructure.

\section{Principaux Résultats Et Discussion}

\section{Caractéristiques Des Répondants}

La population ayant répondu au questionnaire est composée de 433 enseignants de trois cycles, dont une prédominance d'enseignants du cycle primaire avec un pourcentage de $55 \%$ contre $24 \%$ du cycle secondaire collégial et $21 \%$ sont relatifs au cycle qualifiant. Globalement, le questionnaire a touché des tranches variées. Concernant la variable ancienneté, la majorité des enseignants ont une ancienneté inférieure ou égale à 5 ans (36\%), suivi de celle de plus de 20 ans (26\%) alors que ceux qui ont une ancienneté entre 14 et 20 ans représentent $22 \%$ et celle de 9 à 13 ans (9)\%.

Concernant la variable milieu, on note comme que parmi les répondants, $44 \%$ travaillent en milieu rural, $37 \%$ en milieu urbain et $19 \%$ en milieu semi-urbain.

\section{Usage Des TICE}

Sur l'ensemble des répondants, 35\% déclarent utiliser les TICE dont $42 \%$ travaillent dans le milieu rural, $42 \%$ en milieu urbain et $16 \%$ en milieu semi-urbain. Le milieu semble ne pas avoir un effet notable sur l'usage des TICE si on compare le milieu rural et urbain.

Concernant la variable ancienneté, les statistiques révèlent que l'usage des TICE est bien plus accentué chez les nouveaux enseignants puisque $84 \%$ des enseignants utilisant les TICE (oui et parfois) ont une ancienneté inférieure à 5 ans.

$81 \%$ parmi eux ont répondu avoir suivi le module TICE au Centre Régional des Métiers de l'Education et de la Formation (CRMEF). Les rapports institutionnels aux TICE concernant ces derniers résultats seront développés dans l'axe: accompagnement des enseignants dans l'intégration des TICE.

Ce faible pourcentage d'utilisation des TICE en regard des efforts de généralisation déployés sur le plan institutionnel peut-il être expliqué par un manque de:

1. Connaissance du programme GENIE?

2. Accompagnement des enseignants en particulier le renforcement de leurs compétences en matière des TICE?

3. Infrastructure et équipements des établissements scolaires? 


\section{Connaissance du programme GENIE}

Parmi la population étudiée, seulement $25 \%$ déclarent connaître le programme GENIE, $33 \%$ affirment l'ignorer et 27\% estiment le connaître vaguement.

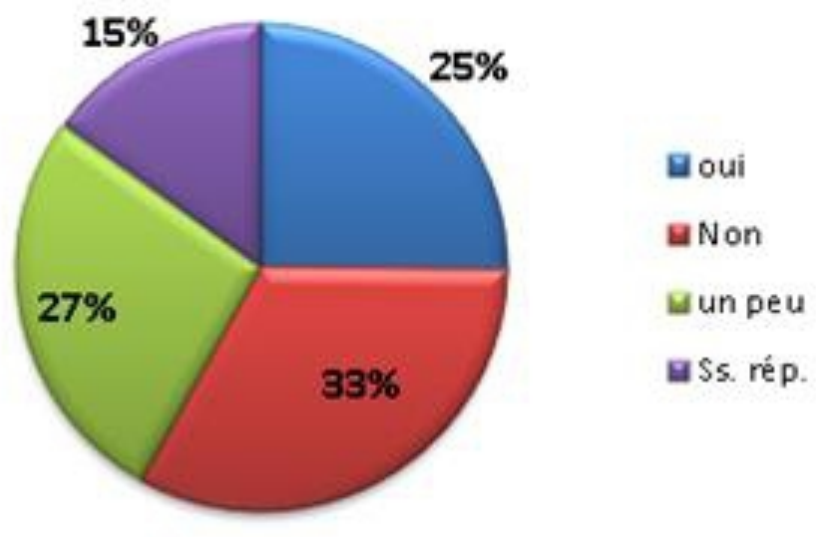

Figure 1: Connaissance du programme GENIE

Ces constats pourraient éventuellement être expliqués par un manque de communication efficace et de vision claire autour dudit programme. D'ailleurs, $30 \%$ des enseignants ont cité ces éléments comme facteurs entravant la mise en œuvre de la stratégie de généralisation des TICE. Or comme souligné par Sierra (2009), le système cognitif en lien avec l'information et la représentation est l'un des obstacles majeurs au changement. L'auteur précise que l'insuffisance des informations permettant la construction de nouvelle représentation du monde, l'incompréhension de la raison du changement ou sa direction et par conséquent son sens entravent son intégration de manière durable.

Or, au niveau institutionnel et suite aux résultats d'arrêt bilan rétrospectif, surtout les aspects liés à la mobilisation et à l'accompagnement des acteurs, plusieurs initiatives ont été entreprises afin de palier à ces problèmes de communication en misant sur la mobilisation des acteurs clés comme les inspecteurs, les directeurs des établissements scolaires et les enseignants (en exercice et stagiaires) et leur implication dans l'opérationnalisation du programme GENIE comme mécanisme d'intégration des technologies d'information. Cependant, les déclarations des enseignants semblent en faveur d'une vision autour du projet, floue ou mal communiquée alors que l'adhésion à une vision est d'une importance capitale dans la conduite de tout changement qui induit des changements dans les croyances individuelles et collectives (attitudes) ainsi que des changements dans les façons de faire (comportements). 
En effet, pour éviter la rupture, il est primordial d'anticiper les risques et les résistances, bien communiquer et faire adhérer autour d'une vision partagée. Cette dernière dépend de la qualité de la phase diagnostique de la situation actuelle (de départ) et du degré d'appropriation de cette vision par les différents acteurs qui en principe devraient participer à sa construction ou impliqués par les initiateurs du changement afin que les acteurs adhèrent au projet sans avoir le sentiment de le subir.

\section{Accompagnement des enseignants}

Concernant la formation des enseignants questionnés dans le domaine des TICE, il parait, d'après leurs déclarations, qu'elle est principalement autonome (52\%). 21\% des enseignants affirment avoir assisté à des formations dans le cadre du programme GENIE tandis que 25\% affirment avoir suivi le module TICE dans les Centres Régionaux des Métiers de l'Education et de la Formation (CRMEF). Ce module a pour but l'acquisition et le perfectionnement des enseignants dans le domaine des TICE afin de les motiver à travailler dans un environnement numérique. D'ailleurs, le pourcentage le plus élevé des usagers des TICE, selon les déclarations des répondants correspond aux lauréats des CRMEF. Quelques cas assez rares ont cité leurs études à l'université et des formations assurées par des associations et/ou par le Centre Marocco-Coréen de la formation (CMCF).

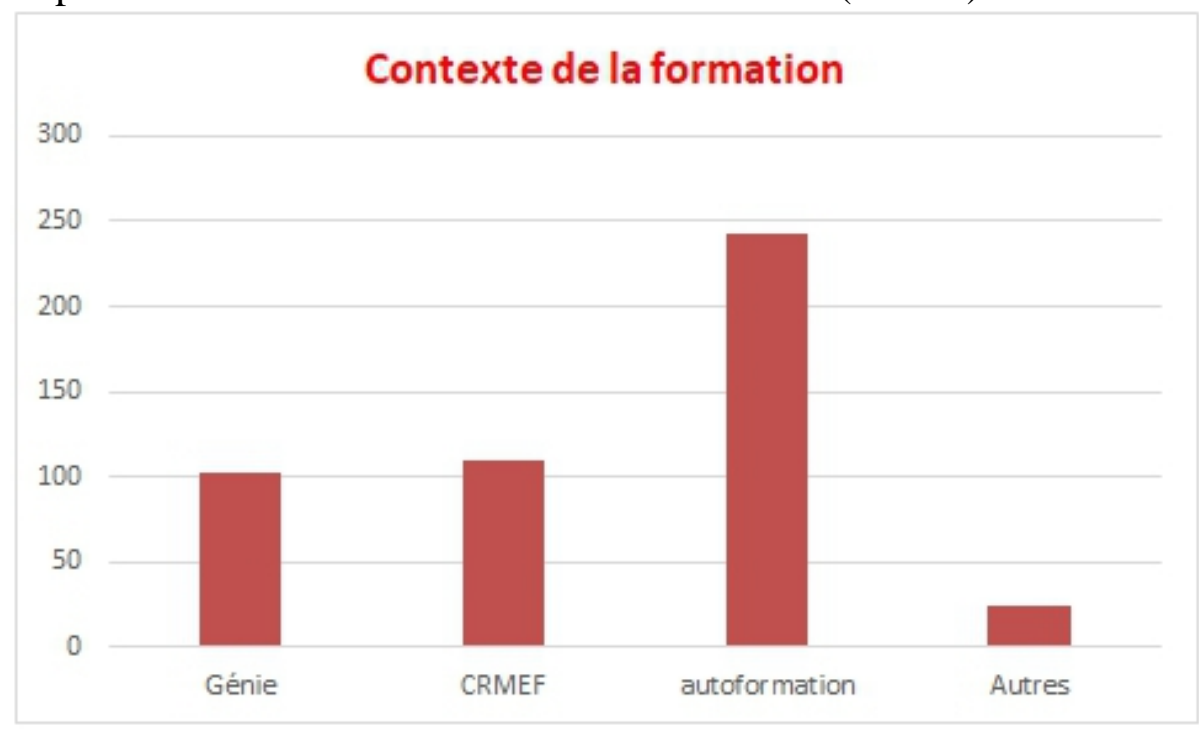

Figure 2: Type de formation

L'analyse des résultats montre une prépondérance à l'autoformation, aussi bien globalement que pour chacune des trois catégories: ceux qui utilisent fréquemment les TICE, ceux qui l'utilisent peu et ceux qui ne l'utilisent pas. Cependant, on note, un pourcentage supérieur chez les 
enseignants qui utilisent les TICE fréquemment ou parfois (44\%) par rapport à celui constaté chez les enseignants qui n'en font pas usage $(8 \%)$. Cela traduit clairement le rôle primordial de la motivation intrinsèque et/ou extrinsèque sur l'appropriation d'un rapport personnel positif envers l'outil et par conséquent sur son usage dans les pratiques enseignantes.

Les statistiques mettent aussi en avant l'importance de l'intégration du module TICE dans la formation initiale des enseignants ainsi que l'utilité des formations assurées dans le cadre du programme GENIE bien qu'elles se limitent, en grande partie, au niveau d'alphabétisation, à la bureautique et à l'usage de l'outil.

Il est donc bien clair que bénéficier des formations GÉNIE favorise visiblement l'usage des TICE dans les pratiques enseignantes, d'où l'urgence d'accélérer davantage la cadence des démultiplications de qualité des formations ainsi que celles des journées de sensibilisation envers l'usage des technologies et des différentes offres de formation.

\section{Infrastructure et équipements des établissements scolaires}

Avant de parler de l'accès ou l'usage de l'équipement relatif au programme GENIE, il est à préciser que les réponses révèlent que l'infrastructure n'est pas toujours au rendez vous. $65 \%$ des questionnés assurent ne pas avoir d'équipement dans leurs établissements, $13 \%$ estiment que l'équipement disponible est insuffisant alors que 14\% seulement affirment sa disponibilité ( $8 \%$ n'y ayant pas répondu). D'ailleurs, le manque d'équipement dans les institutions scolaires constitue pour les répondants, la première entrave à l'utilisation des TICE. Si on considère la variable "milieu", le milieu urbain représente $48 \%$, le rural $37 \%$ et semi urbain $15 \%$.

En ce qui est d'usage des équipements disponibles au sein des établissements, 60\% des usagers des TICE déclarent ne pas y avoir accès contre $61 \%$ pour ceux qui utilisent parfois les TICE. Le risque d'endommager les ordinateurs pose un autre défi pour l'intégration de la technologie. Il parait que les directeurs des établissements n'encouragent pas l'utilisation des ordinateurs par souci de les endommager. Ceci pourrait être expliqué par le fait que les institutions scolaires ne disposent pas de budget propre aux investissements en termes d'équipements et outils technologiques, ou encore en ce qui concerne le capital humain, soit l'embauche de techniciens de maintenance. 


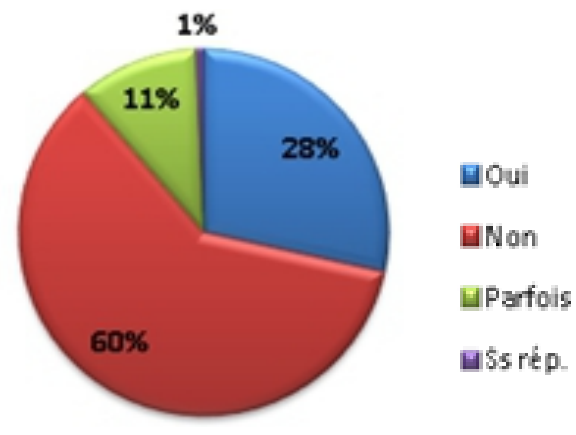

Figure 3: Utilisation de l'équipement du programme GENIE par les enseignants qui affirment utiliser fréquemment les TICE

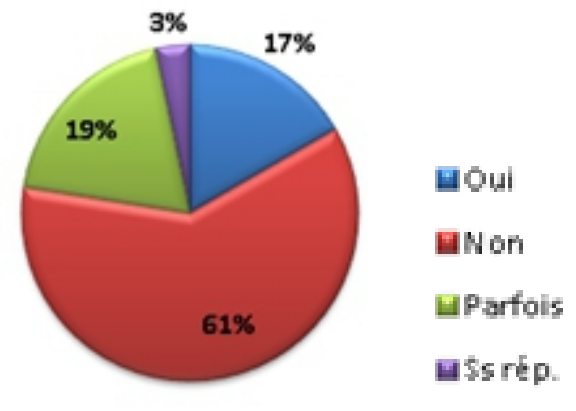

Figure 4: Utilisation de l'équipement du programme GENIE par les enseignants qui affirment utiliser parfois les TICE

Concernant l'Offre de contenus numériques éducatifs et pédagogiques en lien avec les curricula, sur l'ensemble des répondants, 18\% déclarent exploiter les ressources numériques disponibles au niveau de l'établissement alors que la majorité se les procurent via des initiatives personnelles soit en faisant des recherches sur internet (42\%) et ou en les développant personnellement (29\%).

Ce faible taux d'exploitation des ressources numériques dans les établissements scolaires pourrait être expliqué non seulement par la question d'offre mais aussi par la question de sensibilisation et d'information sur le dispositif mis à la disposition des établissements scolaires. Il est à noter toutefois que des efforts ont été déployés dans ce sens et le sont encore, Le ministère de tutelle a procédé à l'acquisition de ressources numériques éducatives variées, un laboratoire national de ressources numériques a été crée en 2009 pour assurer entre autres la veille numérique, la diffusion et l'eformation à l'usage des ressources numériques ainsi qu'un portail dédié aux technologies de l'information et de la communication en éducation, entre autres (GENIE, 2009).

Les résultats obtenus au cours de cette enquête s'alignent globalement avec les travaux cités dans la partie "Revue de la littérature". En effet, le faible usage des TICE relevé dans le cadre de cette étude vient confirmer le constat conclu par l'enquête menée en 2013 à l'académie de Doukkala-Abda (Mastafi, 2013). Elle soutient tout aussi une étude antérieure concernant les attitudes plutôt rétissantes des enseignants envers l'usage des TICE (El Ouidadi, 2012). Pour ce qui est du volet formation de l'étude présente, il vient soutenir les résultats de l'étude menée à l'Académie de Meknès-Tafilalet (Messaoudi, Talbi, 2012), et qui souligne quant à elle des contraintes logistiques concernant les formations elles mêmes ainsi que des difficultés liées au suivi et à l'encadrement des formations tant sur le niveau régional que local. Cette 
dernière étude note aussi l'insuffisance des contenus pédagogiques numériques validés selon les enseignants, sujets de cette expérimentation, chose que confirme aussi l'enquête menée dans le cadre de ce travail dans son volet infrastructure et équipement.

Or la communication et la clarté des enjeux, l'accompagnement, la formation des acteurs ainsi que l'appui matériel et logistique bref l'instauration d'un climat propice au changement sont essentiels pour garantir l'adhésion à tout projet (Kotter, 1996; Minvielle et Contandriopoulos , 2004).

\section{Conclusion}

Les résultats de cette étude montrent d'après les déclarations des enseignants ayant répondu au questionnaire que la majorité ne connaît pas ou peu le programme GENIE. Or, comme il est stipulé par Kotter (1996), la création d'un climat propice au changement est une étape cruciale dans le processus d'instauration de toute innovation. Concernant le renforcement des compétences des acteurs clé dans la mise en œuvre du changement dans les pratiques enseignantes escomptées, la majorité des répondants déclarent bénéficier d'auto-formation alors que plusieurs offres existent en présentielle ou à distance. Il y'a un vrai problème de communication sur diverses offres dédiées aux technologies d'information et de communication telles que le portail MOOC Genie tice, ComPractice (ComPractice,2019), PDP-TICE (PDPTICE,2013), Collab (Collab). Ces offres semblent désarticulées alors que la finalité est commune. Ce qui est attrait aux moyens et équipements nécessaires à l'implantation d'une culture numérique, il semble que cette composante fait défaut selon les déclarations des enseignants. La majorité des usagers des TICE a recours à des initiatives personnelles. Le même constat pour ce qui est infrastructure.

Quelle que soit l'ampleur des efforts et des moyens déployés, la mise en place des réformes et leur réussite sont tributaires de la mise en œuvre de stratégies de conduite du changement favorisant une dynamique mobilisatrice autour de l'éducation en général et de l'intégration des TICE en particulier. La sensibilisation, la communication, la responsabilisation, la révision et l'actualisation de la formation en termes de logistique, d'encadrement, d'accompagnement et de contenus sont nécessaires et indispensables pour l'avancement de la généralisation des technologies d'information et de communication.

Enfin, pour que les réformes entreprises aient de l'incidence sur les pratiques professionnelles, les apports de savoirs et de savoir-faire devraient être pertinents aux actions de changement ciblées et le travail des agents de changement, qui constituent le personnel d'encadrement de proximité, de qualité. 


\section{References:}

1. Abouhanifa, S; Drissi, M; , Kabbaj, M et Talbi, M. ( 2009). Programme GENIE au Maroc: TICE et développement professionnel. Disponible sur http://revue.sesamath.net/spip.php?article233

2. Ahaji, K et El Hajjami, A. (2010). Le concours des enseignants innovants : Un bon exemple pour la promotion des innovations TICE. Disponible sur https://www.epi.asso.fr/revue/articles/a1010f.htm

3. Basque, J. (2006) . Une réflexion sur les fonctions attribuées aux TIC en enseignement universitaire. Disponible sur: https://edutice.archives-ouvertes.fr/edutice-00086399/document

4. Benheddi, R. (2015). les TICE à l'école rurale au Maroc, échec ou une réussite?. Disponible sur: ww.google.com/amp/edupronet.com/lestice-a-l-ecole-rurale-au-maroc-echec-ou-succes/amp/

5. Bilans du projet E1P10. (2009-2010). Disponible sur http://www.taalimtice.ma/fr/taxonomy/term/9

6. Champagne, F. (2002). La capacité de gérer le changement dans les organisations de santé. Gouvernement du Canada: Commission Romanov, Etude n³6. ISBN 0-662-87980-5.

7. CCME-Lancement Du Nouveau Réseau "marwan" Pour L'amélioration De La Qualité Du Réseau Dans Les Universités (23.12.2009). Disponible sur sur https://www.ccme.org.ma/fr/actualites/3826

8. Collab. Disponible sur http://collab.men.gov.ma

9. ComPractice (2019) . Disponible sur http://compractice.men.gov.ma/

10. Cros, F. (2004). L'innovation scolaire aux risques de son évaluation. Paris : L'Harmattan,. 208 pages. Savoir et formation. ISBN : 2-74756812-1.

11. CSEFRS. (n.d.). Vision Stratégique de la réforme 2015-2030 : POUR UNE ÉCOLE DE L'ÉQUITÉ, DE LA QUALITÉ ET DE LA PROMOTION. Disponible sur https://www.csefrs.ma/publications/vision-strategique-de-lareforme/?lang $=\mathrm{fr}$

12. El Madhi, Y; Chiahou, B; Belghiti, D; El Kharrim, Kh et El Halouani, H. (2014). Contraintes liées à l'intégration des TIC dans l'enseignement des Sciences de la Vie et de la Terre au Maroc. European Scientific Journal, 10 (34), 143-153.

13. El Ouidadi, O. (2012). Etat des lieux et enjeux de l'implémentation des TICE dans le système scolaire marocain : profils des enseignants et étude d'impact en SVT. Thèse de doctorat, laboratoire de recherche LADIPEC, Faculté des sciences Dhar El Mahraz, Université Sidi Mohamed Ben Abdellah, Fès, Maroc. 
14. Hamon, D. (2012). Le rapport des enseignants de l'école élémentaire aux TICE : adaptabilité et distance critique. Adjectif Analyses Recherches sur les TICE. Disponible sur http://www.adjectif.net/spip/spip.php?article194

15. Isabelle, C; Lapointe, C et Chiasson, M. (2002). Pour une intégration réussie des TIC à l'école : De la formation des directions à la formation des maîtres. Revue des sciences de l'éducation. 28 (2), 325343. Disponible sur https://doi.org/10.7202/007357ar

16. GENIE. (2009) . Programme GENIE, Premier bilan de la stratégie 2009-2013. Disponible sur http://www.taalimtice.ma/fr/node/224

17. Guide général TICE. (version septembre 2014). Disponible sur http://www.taalimtice.ma/sites/default/files/IMAGES_ACTUALITE S/2eme-Ver-final-Guides-Pedag-TICE/Guide-TICE-

General_2\%20Ver_2-Septembre\%202014.pdf

18. Janner, M; Lescouarch, L. (2013). Représentations du changement chez les enseignants dans le champ scolaire en France et accompagnement d'équipes d'école. Spirale|Revue de recherche en éducation $\quad \mathrm{N}^{\circ} \quad 51, \quad 113-132 . \quad$ Disponible sur https://www.persee.fr/doc/spira_0994-3722_2013_num_51_1_1076

19. Kotter, J-P. (1996). Leading change. Boston: Harvard Business School Press (HBS). ISBN 978-0-87584-747-4

20. Mastafi, M. (2013). Intégration et usage des TIC dans le système éducatif marocain: Attitudes des enseignants de l'enseignement primaire et secondaire. Adjectif Analyses Recherches sur les TICE. Disponible sur www.adjectif.net/spip/spip.php?article228

21. Messaoudi, F et Talbi, M. (2012). Regard sur le déploiement de la stratégie nationale GENIE. Association EPI. Disponible sur www.epi.asso.fr

22. Ministère de l'Industrie, du Commerce et des Nouvelles Technologies (MICNT). (2009). Maroc Numeric 2013, Stratégie Nationale pour la Société de l'Information et de l'Économie Numérique. Disponible sur http://www.egov.ma/sites/default/files/Maroc\%20Numeric\%202013. pdf

23. Ministère de l'Education Nationale (MEN). (2019). Encourager l'excellence, l'innovation et la recherche scientifique. Disponible sur https://www.men.gov.ma/Fr/Pages/CNEF_espace3-5.aspxMintzberg, H et Wisley, F. (1992). "Cycles of Organization Change”, Strategic Management Journal, Hiver 1992, N 13 édition spéciale, pp. 39-59.

24. Minvielle, É; Contandriopoulos, A-P. (2004). La conduite du changement, quelles leçons tirer de la restructuration hospitalière? Lavoisier | Revue française de gestion. 3(150), 29-53. 
Disponible sur https://www.cairn.info/revue-francaise-de-gestion2004-3-page-29.htm

25. MOOC Genie tice. (n.d.). Disponible sur le portail : http://www.taalimtice.ma/formation/modules/1967/\%D9\%85\%D9\%8 6\%D8\%B5\%D8\%A9-

\%D8\%A7\%D9\%84\%D8\%AA\%D9\%83\%D9\%88\%D9\%8A\%D9\%8

6-mooc-genie-tice

26. Moscovici, S. (1991). Psychologie des minorités actives. Paris: PUF, 3e édition, 275 pages. Collection: Sociologies, première édition : 1979. Disponible sur http://dx.doi.org/doi:10.1522/030149037

27. Nafidi, Y; Alami, A; Zaki, M; El Batri, B; Elazami, H-M et Afkar, H. ( Janvier 2018). L'intégration Des TIC Dans L'enseignement Des Sciences De La Vie Et De La Terre Au Maroc: État Des Lieux Et Défis À Relever. European Scientific Journal, 14, 1857 - 7881. Disponible sur http://dx.doi.org/10.19044/esj.2018.v14n1p97

28. Nimier, J. (2016). Les sept conditions d'un changement réussi. In André Giordan. Le changement en éducation. France, École changer de cap. Septembre 2016, 54- 58. Disponible sur http://www.ecolechangerdecap.net/IMG/pdf/dossier_changement.pdf

29. PDP TICE. Guide du formateur. (2013). Disponible sur http://www.taalimtice.ma/formation/modules/1957/pdp-tice--والتكوين والإنماء_المهني

30. Plan d'action pour l'axe «formation» du programme GENIE. (2006). Disponible sur http://www.taalimtice.ma/fr/node/15

31. Rapport d'évaluation de GENIE 1. (Juillet 2008). Disponible sur http://www.taalimtice.ma/fr/node/45

32. Rapport sur l'Analyse des besoins et des priorités en matière de ressources pédagogiques numériques ; Programme GENIE. (Juin 2008). Disponible sur http://www.taalimtice.ma

33. Résumé du rapport d'évaluation interne de l'usage des technologies d'information et de communication dans les pratiques pédagogiques. (2016-2017).

Disponible sur http://www.taalimtice.ma/sites/default/files/ONUTICE/PIECESJOINTES/fiche_technique_evaluation_interne_-_2016-2017.pdf

34. Résumé de l'étude de l'évaluation interne de l'usage des technologies d'information et de communication dans les pratiques pédagogiques. (2014-2015).

Disponible sur http://www.taalimtice.ma/sites/default/files/ONUTICE/PIECESJOINTES/resume_evaluation_interne_2014-2015.pdf

35. Résumé du rapport de l'évaluation interne de l'étendue de l'usage des technologies d'information et de communication dans les pratiques 
pédagogiques. (Avril 2012- Avril 2013). Disponible sur http://www.taalimtice.ma

36. Sandholtez, J-H; Ringstaff, C et Dwyer, D-C. (1997). La classe branchée. Enseigner à l'ère des technologies. Paris: CNDP, 1997. 210 pages. Dossiers de l'ingénierie éducative. ISBN 978-2240004963, Cité par Isabelle et al (2002).

37. Sidir, M; Benchenna, A. (2008). Du recours aux TICE en temps de crise? Le cas des universités marocaines. Disponible sur https://ds.revuesonline.com/gratuit/DS6_2_05_Sidir.pdf

38. Stratégie pour le développement de contenus éducatifs basés sur les TIC (Version du 23/02/2006). Disponible sur http://www.taalimtice.ma/fr/node/15

39. UNESCO. (2017). Stratégie Maroc Digital 2020. Disponible sur $<$ https://en.unesco.org/creativity/periodic-reports/measures/strategiemaroc-digital-2020> 